\section{The fuss over lipo "fuss"cin: not all autofluorescence is the same}

\author{
B. Sainz Jr., ${ }^{1,2}$ I. Miranda-Lorenzo, ${ }^{1}$ \\ C. Heeschen ${ }^{1,3}$
}

'Stem Cells \& Cancer Group, Molecular

Pathology Programme, Autonoma

University of Madrid, Spain

2Department of Preventive Medicine,

Public Health and Microbiology,

Autonoma University of Madrid, Spain

${ }^{3}$ Centre for Cancer, Stem Cells \& Ageing,

Barts Cancer Institute, Queen Mary

University of London, UK

\begin{abstract}
Since the first description of cellular autofluorescence over a century ago, we have now come to appreciate that autofluorescence should not be discarded as a biological artifact but embraced as a biological phenomenon with potentially important cellular relevance. Indeed, cellular and tissue autofluorescence has been attributed to a spectrum of unrelated molecules such as porphyrins, vitamins (vitamin A, riboflavin, thiamine), structural proteins, lipofuscin and ceroid pigments. We have recently shown that freshly isolated epithelial cancer stem cells (CSCs) bear autofluorescent vesicles in the cytoplasm. Our studies definitively prove that riboflavin and not lipofuscin is the source of autofluorescence in CSCs as the inhibition of ATP and not autophagy elimiwe definitively show that riboflavin (vitamin $\mathrm{B}_{2}$ ) is the underlying causative agent of autofluorescence in epithelial CSCs, Prof. Di Guardo takes the liberty to dispute our exhaustive investigation by proposing lipofuscin as an alternative source and additionally raises the notion that not considering lipofuscin and lipofuscin-like compounds among the biomolecular substrates potentially-putatively responsible for the autofluorescence specifically detected in epithelial CSCs could be regarded as a conceptual error leading to a methodological slip. To avoid confusing the reader, we here address Prof. Di Guardo's concern and explain why his claim is unprecedented and incorrect.

\section{Intracellular autofluorescence and spectral profiles} nates CSC autofluorescence, that the ATPdependent transporter ABCG2, for which riboflavin is a substrate, is overexpressed in autofluorescent CSCs and co-localizes with the membrane of intracellular autofluorescent vesicles, the ABCG2-specific inhibitor Fumitremorgin $\mathrm{C}$ reversibly eliminates CSC autofluorescence, riboflavin is a substrate for ABCG2, and only the addition of riboflavin to vitamin-deprived CSC cultures is capable of restoring autofluorescence. Thus, the sum of these data unequivocally supports the conclusion that the source of CSC autofluorescence is the vitamin riboflavin.

\section{Introduction}

We appreciate Prof. Di Guardo's interest in our recent work published in Nature Methods where we describe that a subpopulation of cancer stem cells (CSCs) present in numerous epithelial solid tumors contains intracellular autofluorescent vesicles. These allow for the prospective isolation, purification and biological characterization of CSCs. ${ }^{1}$ We would like to take this opportunity, however, to formally address specific issues raised by Prof. Di Guardo regarding the source of the autofluorescent phenotype we describe and characterize in our study. ${ }^{2}$

Since the first description of cellular autofluorescence over a century ago, we have now come to appreciate that autofluorescence should not be discarded as a biological artifact but embraced as a biological phenomenon with potentially important cellular relevance. Indeed, cellular and tissue autofluorescence has been attributed to a spectrum of unrelated molecules such as porphyrins, vitamins (vitamin A, riboflavin, thiamine), structural proteins, lipofuscin and ceroid pigments. ${ }^{3}$ While

Autofluorescence in cells and tissues can be detected due to the presence of intrinsic biomolecules acting as endogenous fluorophores, with favourable spectral properties and quantum efficiency (excitation/emission ranges within the blue region where most endogenous fluorophores emit). ${ }^{3}$ Specifically, the auto-fluorescence in the cytoplasm of epithelial CSCs has a distinct green spectral profile (excitation and emission 480-490 $\mathrm{nm}$ and 530$540 \mathrm{~nm}$, respectively) and can be excited only with a standard blue laser, but not with yellowgreen (e.g., $561 \mathrm{~nm})$ or red (e.g., $640 \mathrm{~nm})$ lasers. Riboflavin has a near identical spectral profile (excitation and emission 480-490 nm and 530-540 nm, respectively), while lipofuscin and lipofuscin-like lipopigments have a much broader spectral profile with excitation and emission wavelengths of 400-500 nm and 480-700 nm, respectively (Figure 1). Thus, the autofluorescence of lipofuscin or lipofuscinlike lipopigments covers the yellow-red region, depending on the variability of its composition
Correspondence: Dr. Christopher Heeschen, Centre for Cancer, Stem Cells \& Ageing, Barts Cancer Institute, Queen Mary University of London, Charterhouse Square, London EC1M 6BQ, UK. E-mail: c.heeschen@qmul.ac.uk

Conflict of interest: the authors declare that no conflict of interest exists for the presented study.

Keywords: Cancer stem cells, autofluorescence, epithelial cancers.

Received for publication: 25 February 2015.

Accepted for publication: 6 March 2015

This work is licensed under a Creative Commons Attribution NonCommercial 3.0 License (CC BYNC 3.0).

CC Copyright B. Sainz Jr. et al., 2015

Licensee PAGEPress, Italy

European Journal of Histochemistry 2015; 59:2512 doi:10.4081/ejh.2015.2512

(e.g., proteins, lipids, carotenoids and metals). ${ }^{4}$ Lipofuscins have been shown to consist, in part, of undigested material (e.g., lipids and covalently cross-linked proteins) remaining from phagocytosis and/or autophagy and therefore they can be occasionally observed in the cell cytoplasm as intracytoplasmic granules $^{5-7}$ In our work, we specifically excluded a link between CSC autofluorescence and autophagy, and CSC autofluorescent vesicles also did not co-localize with lipid droplets or lysosomes, as demonstrated by co-staining with Nile red or LysoTracker, respectively. ${ }^{1}$ In addition, autofluorescence was not observed in paraffin sections of pancreatic tumors (data not shown), which is in contrast to lipofuscin autofluorescence, which is not lost when tissues are formalin fixed and paraffin embedded, likely due to its non-degradable makeup. ${ }^{8}$ While the spectral data and the aforementioned experimental observations alone would argue that the source of the CSC autofluorescence we observe in freshly digested or cultured tumors is not lipofuscin, our ABCG2-specific studies leave no doubt that riboflavin and not lipofuscin is the source of autofluorescence in CSCs. Specifically, we show that inhibition of ATP and not autophagy eliminates CSC autofluorescence, that the ATP-dependent transporter ABCG2 is overexpressed in autofluorescent CSCs and co-localizes with the membrane of intracellular autofluorescent vesicles, the ABCG2-specific inhibitor Fumitremorgin $\mathrm{C}^{9}$ reversibly eliminates CSC autofluorescence, riboflavin is a substrate for ABCG2,${ }^{10}$ and only the addition of riboflavin to vitamin-deprived CSC cultures is capable of restoring autofluorescence. Thus, the sum of these data unequivocally supports the conclu- 

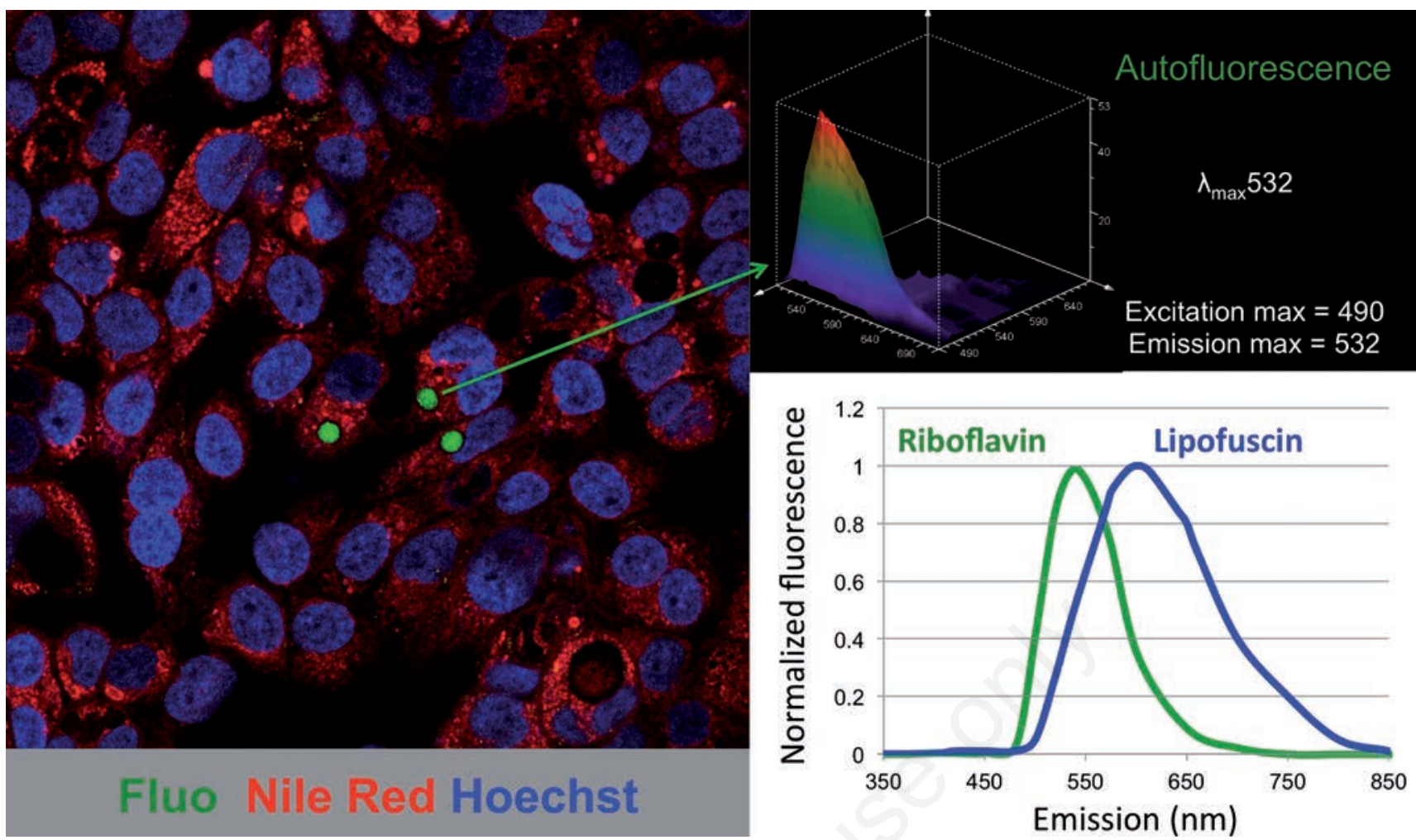

Figure 1. Autofluorescence in epithelial cancer stem cells. Confocal image of cancer cells derived from primary pancreatic cancer tissue showing a subset of cells with autofluorescent cytoplasmic vesicles (left). Spectral analysis of the autofluorescence demonstrated an emission maximum at $532 \mathrm{~nm}$ (upper right). Representative emission spectra of riboflavin and lipofuscin (lower right). Fluo, autofluorescence; Nile Red, lipid droplets; Hoechst, nuclear staining.

sion that the source of CSC autofluorescence is the vitamin riboflavin.

\section{Concluding remarks}

We thank the journal for sharing Prof. Di Guardo's opinion, and we hope that our response provides evidence-based clarity to support our claim that riboflavin and not lipofuscin is the source of autofluorescence in epithelial CSCs and that no methodological slip has been made. These minor quibbles, however, should not distract from the clear consensus of all the authors that autofluorescence in CSCs represents an important advance in our understanding of these biologically important cells in the context of cancer and chemoresistance.

\section{References}

1. Miranda-Lorenzo I, Dorado J, Lonardo E, Alcala S, Serrano AG, Clausell-Tormos J, et al. Intracellular autofluorescence: a biomarker for epithelial cancer stem cells. Nat Methods 2014;11:1161-9.

2. Di Guardo G. Lipofuscin, lipofuscin-like pigments and autofluorescence. Eur $\mathrm{J}$ Histochem 2015;59:2485.

3. Croce AC, Bottiroli G. Autofluorescence spectroscopy and imaging: a tool for biomedical research and diagnosis. Eur J Histochem 2014;58:2461.

4. Wolman M. Lipid pigments (chromolipids): their origin, nature, and significance. Pathobiol Annu 1980;10:253-67.

5. Brunk UT, Jones CB, Sohal RS. A novel hypothesis of lipofuscinogenesis and cellular aging based on interactions between oxidative stress and autophagocytosis. Mutat Res 1992;275):395-403.
6. Jung T, Bader N, Grune T. Lipofuscin: formation, distribution, and metabolic consequences. Ann N Y Acad Sci 2007;1119: 97-111.

7. Sulzer D, Mosharov E, Talloczy Z, Zucca FA, Simon JD, Zecca L. Neuronal pigmented autophagic vacuoles: lipofuscin, neuromelanin, and ceroid as macroautophagic responses during aging and disease. $\mathrm{J}$ Neurochem 2008;106:24-36.

8. Terman A, Brunk UT. Lipofuscin. Int J Biochem Cell Biol 2004;36:1400-4.

9. Rabindran SK, Ross DD, Doyle LA, Yang W, Greenberger LM. Fumitremorgin C reverses multidrug resistance in cells transfected with the breast cancer resistance protein. Cancer Res 2000;60:47-50.

10. van Herwaarden AE, Wagenaar E, Merino G, Jonker JW, Rosing H, Beijnen JH, et al. Multidrug transporter ABCG2/breast cancer resistance protein secretes riboflavin (vitamin B2) into milk. Mol Cell Biol 2007;27:1247-53. 